\title{
Civilisations
}

Revue internationale d'anthropologie et de sciences

humaines

55 | 2006

Confrontations et alliances dans les Amériques autochtones

\section{Droits autochtones amazoniens et droit officiel équatorien : une opposition culturelle?}

Le cas des Runa et des Shiwiars

\section{Barbara Truffin}

\section{OpenEdition}

\section{Journals}

Édition électronique

URL : http://journals.openedition.org/civilisations/262

DOI : 10.4000 /civilisations. 262

ISSN : 2032-0442

\section{Éditeur}

Institut de sociologie de l'Université Libre de Bruxelles

Édition imprimée

Date de publication : 1 octobre 2006

Pagination : 143-162

ISBN : 2-87263-10-4

ISSN : 0009-8140

\section{Référence électronique}

Barbara Truffin, «Droits autochtones amazoniens et droit officiel équatorien : une opposition culturelle? », Civilisations [En ligne], 55 | 2006, mis en ligne le 01 octobre 2009, consulté le 19 avril 2019. URL : http://journals.openedition.org/civilisations/262 ; DOI : 10.4000/civilisations.262 


\title{
Droits autochtones amazoniens et droit officiel équatorien: une opposition culturelle?
}

\author{
Le cas des Runa et des Shiwiars ${ }^{1}$
}

\section{Barbara TRUFFIN}

Résumé : Le paradoxe de la reconnaissance constitutionnelle de droits aux peuples autochtones réside dans la distinction qu'elle présuppose entre les normativités autochtones et le droit étatique. "Peut-on différencier les droits autochtones amazoniens $d u$ droit équatorien officiel en fonction de critères culturels? "1. Cet article propose une réflexion sur cette question. L'analyse de deux cas tirés de quotidiens amazoniens, shiwiar et runa, permet d'établir qu'il est difficile de répondre à cette question de manière affirmative (1) ou négative (2). Pour sortir de ce faux-débat, on interrogera la formulation de cette distinction entre droits autochtones et droit officiel et ce qu'elle suppose en terme de constitutionnalisation (3). Cette porte de sortie invite à penser autrement les relations entre différents « droits » et différentes « cultures » en Amazonie équatorienne (4).

Mots-clés : anthropologie juridique, droits des peuples autochtones, constitutionnalisation, Amazonie équatorienne.

Summary: The distinction between indigenous law and State law constitutes a paradox for the constitutional recognition of indigenous rights. "Is it possible to differentiate Amazonian indigenous laws from Ecuadorian State law on the basis of cultural criteria?" This is the issue discussed in the present contribution. The analysis of two cases drawn out runa and shiwiar daily lives conduct to the conclusion that it is impossible to give an affirmative (1) or a negative (2) answer to the question raised by the constitutional text. In order to escape from an impossible debate, we choice to interrogate the constitutional formulation of the distinction between "systems" of state and indigenous laws (3). This way out should allow us to reflect, in a renewed manner, on the relationships between different "rights" and different "cultures" in the Ecuadorian Amazon (4).

Keywords: legal anthropology, indigenous peoples rights, constitutionalization, Ecuadorian Amazon.

1. Remerciements : Les recherches sur lesquelles se base cet article n'auraient pas pu être menées sans le soutien du FNRS que je remercie. Elles ont également bénéficié de l'accueil du Centre d'anthropologie culturelle et de celui du Centre de droit comparé et d'histoire du droit de l'ULB et de leurs membres. Ma gratitude pour les dirigeants shiwiar et quichua, qui ont patiemment travaillé avec moi, est immense. 
T e questionnement sur les relations entre les droits étatiques et les droits Lautochtones s'étend bien au-delà des problèmes rencontrés par les Runa et les Shiwiar d'Amazonie équatorienne sur lesquels cet article porte ${ }^{2}$. La délimitation et la reconnaissance de droits autochtones se discutent à l'échelle mondiale. Ces questions sont à l'ordre du jour de nombreuses réunions internationales et nationales consacrées aux «droits des peuples autochtones ». Ce sujet d'interrogation est loin d'être purement théorique et institutionnel. Le statut culturel et juridique autochtone fonctionne aujourd'hui comme une « monnaie » d'échange pour les représentants de nombreux groupes. Il oriente leurs relations avec les institutions étatiques et les organisations non gouvernementales.

Nous proposons d'examiner ces relations entre droit officiel et droits autochtones à la lumière d'une expérience de terrain avec des dirigeants indiens de deux sociétés amazoniennes d'Equateur, les Runa et les Shiwiar. Les familles de ces deux groupes ethniques voisins sont établies dans la province du Pastaza ${ }^{3}$. En terme démographique, l'ensemble runa correspond approximativement à une dizaine de milliers de personnes, tandis qu'on dénombre moins d'un demi-millier de personnes parmi les familles shiwiar réparties autour de neuf centres résidentiels (fig.1).

Bien que certains de leurs membres soient trilingues ${ }^{4}$ et que les groupes de parenté intermariés ne soient pas rares, on peut différencier ces ensembles voisins sur base de critères linguistiques, culturels et sociaux. Les Runa parlent le quichua amazonien, alors que les Shiwiar utilisent une variante jivaro proche de l'achuar.

2. Ces recherches de terrain ont été conduites auprès de deux organisations amazoniennes dans la province du Pastaza en 2001 (septembre à décembre) et en 2002 (avril à décembre). Les enquêtes se sont concentrées sur le travail des dirigeants et de leurs conseillers techniques dans les locaux urbains de ces organisations à Puyo. Les dirigeants et les conseillers techniques sont des interlocuteurs privilégiés pour aborder les questions juridiques. Ils sont confrontés en première ligne à la différenciation culturelle des « droits autochtones » dans leurs actions politiques. La constitution des données a donc été fortement influencée par l'expérience de ces nouveaux spécialistes politiques, au détriment des perspectives d'autres membres des groupes runa et shiwiar. Il nous semble que cette disproportion n'est pas de nature à invalider les résultats de cette recherche. Les raisons qui motivent cette position méthodologique tiennent au genre de divergences qui acquiert une importance politique au sein de ces deux groupes autochtones. En règle générale, ce type de conflits ne porte pas sur la nécessité d'avoir et de revendiquer des « droits » ou des organisations politiques autochtones. Les différends liés aux nouvelles institutions politiques portent presque exclusivement sur deux types de contestations : la détermination des limites du groupe représenté d'une part, et les mérites des dirigeants que les assemblées désignent et révoquent d'autre part. Le travail des dirigeants et des techniciens, artisans de ces processus d'institutionnalisation, constitue donc un matériel de données particulièrement significatif pour l'analyse de ces processus récents.

3. La province du Pastaza, institutionnalisée dans les années 60, comprend environ $25000 \mathrm{~km}^{2}$ de forêt tropicale et $5000 \mathrm{~km}^{2}$ d'une bande de terres déboisées à l'ouest. Ce déboisement trouve son origine dans la colonisation agraire des quarante dernières années. A l'ouest, la province est parcourue par des routes qui la relient aux autres provinces amazoniennes et aux plateaux andins. Elle est parsemée de petites agglomérations. Le chef-lieu de la province est la petite ville de Puyo. On y pratique l'élevage et une agriculture de produits exotiques (naranjilla, canne à sucre) orientés vers les marchés locaux et nationaux. (Garí $2001: 3$ ).

4. L'espagnol, teinté d'un accent amazonien, constitue la troisième langue parlée dans ces groupes. 


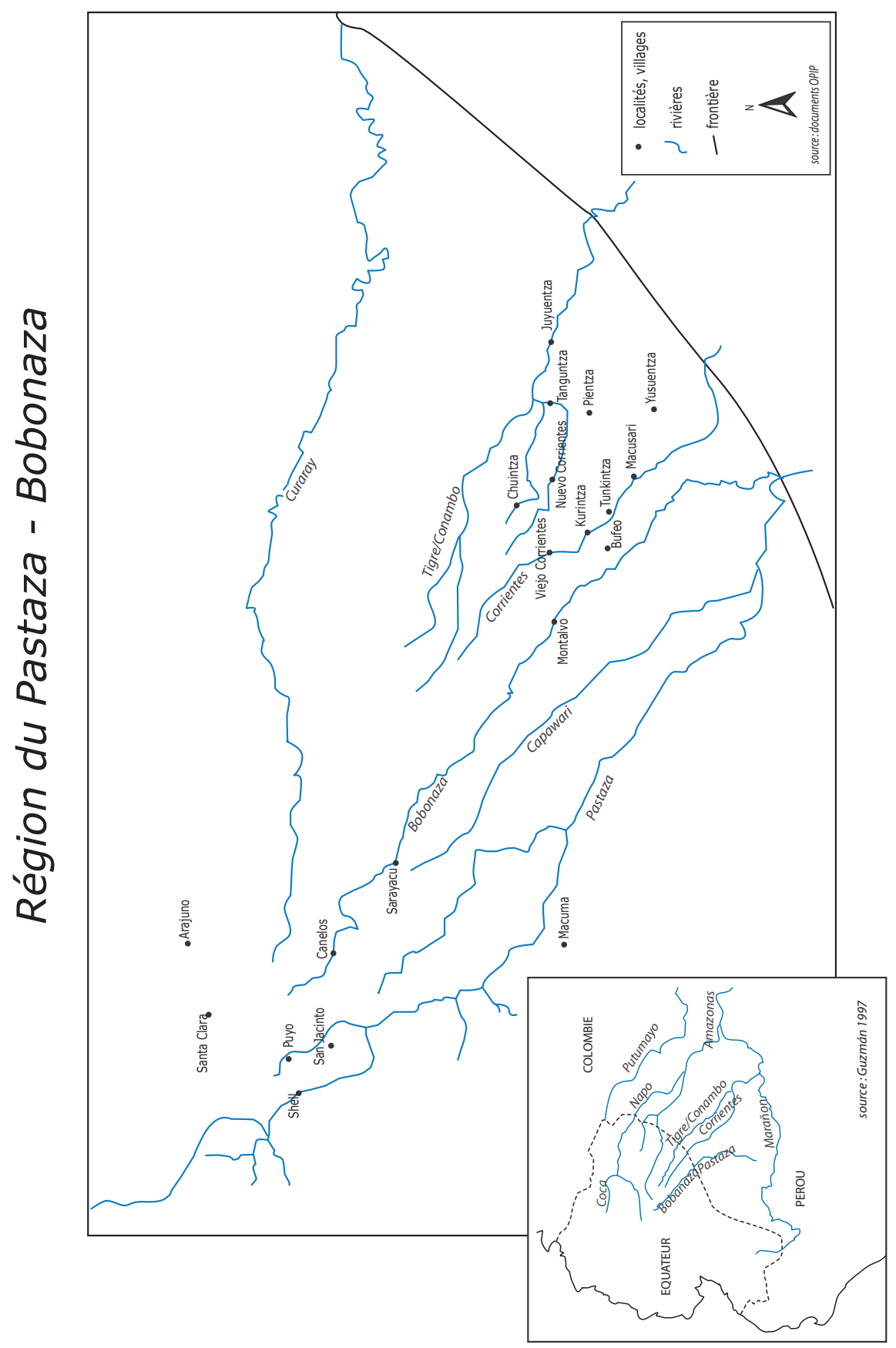

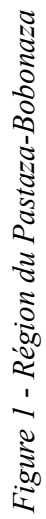

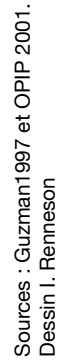


De manière très schématique, on dira que les familles runa résident à proximité des petites bourgades amazoniennes et dans un environnement relativement détérioré par rapport à celui de la forêt où vivent les Shiwiar, non loin de la frontière avec le Pérou ${ }^{5}$. Les activités quotidiennes des Shiwiar et des Runa impliquent des paradigmes chamaniques et d'interprétation onirique complexes et développés (Bilhaut 2003; MacDonald 1999; Taylor 1981; Whitten 1976, 1985). Les relations avec les entités intervenant dans les pratiques de chasse pour les hommes et des travaux au jardin pour les femmes ne diffèrent pas beaucoup entre ces voisins qui peuvent se reconnaître dans une indianité générique face aux « autres » occupants des espaces amazoniens de l'Equateur ${ }^{6}$.

Leurs modalités de résidence sont de plus en plus similaires depuis que les familles shiwiar ont décidé, il y a quelques décennies, de regrouper leurs maisons autour des petites pistes d'avionnettes que les missionnaires évangéliques et les militaires équatoriens ont ouvertes en forêt. Toutefois les communautés territoriales runa sont plus nombreuses, plus densément peuplées et se sont constituées il y a plus longtemps que les centres shiwiar.

La constitution des frontières ethniques est souple. Le domaine des relations familiales est spécialement investi pour exprimer et souligner ces distinctions. La polygynie et les guerres de vendetta, bien qu'elles ne soient plus toujours ouvertement pratiquées, restent des institutions valorisées dans le discours familial shiwiar. Pour leur part, les Runa se gaussent mi-amusés, mi-envieux, de ces relations multiples et jurent avoir renoncé aux guerres « sauvages ». Quoi qu'il en soit de l'objectivité de ces différences, des logiques communes alimentent ces ethos culturels différenciés. L'honneur du groupe de référence et la construction culturelle de figures d'hommes et de femmes forts et valeureux [sinchi en quichua; kakaram en achuar] (Descola 1993a; Whitten 1985) sont sans conteste des thèmes dominants et presque obsédants dans la vie quotidienne de l'ensemble de ces familles.

La concentration et l'intensification démographique des familles runa et shiwiar dans des écosystèmes fragiles, perturbés par l'urbanisation et l'industrialisation pétrolière rapides et mal contrôlées de la région amazonienne, constituent des défis de taille pour ces familles. L'Amazonie équatorienne connaît ces dernières décennies un accroissement de conflits aigus, de plus en plus insolubles. Les enjeux pétroliers alimentent une série de conflits institutionnels mais également privés. Le « développement» de la région amazonienne a attiré des petits agriculteurs andins en quête de travail et de terres mais n'a pas tenu ses promesses de prospérité.

L'accès aux ressources naturelles est dès lors un thème particulièrement conflictuel dans cette région en mutation. Dans ce contexte mouvant, les leaders runa et shiwiar

5. Il s'agit là d'une simplification. Les communautés runa étant nettement plus nombreuses que celles des Shiwiar, elles sont aussi réparties sur une échelle géographique plus étendue. En tout état de cause, quelques communautés runa vivent dans la région frontalière peruano-équatorienne et sont donc géographiquement plus proches des familles shiwiar que des communautés runa de l'amont.

6. La construction de cette frontière ethnique ne peut occulter le fait que d'autres occupants - les militaires équatoriens et les résidents américains évangéliques mis à part - proviennent de la petite paysannerie indigène et métisse des régions andines. Ils sont également marginalisés et vivent dans des situations précaires. On ne peut que déplorer l'injustice qu'implique une protection juridique différenciée en fonction de critères d'authenticité culturelle. Nous n'y reviendrons pas dans le cours de cet article. Nous nous contenterons de souligner les problèmes généraux impliqués par la différenciation culturelle des droits autochtones dans le processus de constitutionnalisation équatorien. 
se sont « organisés" dans de nouvelles structures institutionnelles, les organisations indigènes. Au niveau national, ils «luttent» avec les dirigeants d'autres peuples autochtones andins et amazoniens pour que leurs " territoires », leurs « droits » et leurs manières de faire soient garantis et reconnus. La Constitution équatorienne a été modifiée en ce sens en $1998^{7}$. La garantie des droits des peuples autochtones est présentée, dans le texte constitutionnel équatorien, comme impliquant une différenciation culturelle des droits autochtones et étatiques.

Les enjeux de la délimitation de droits autochtones et de leurs relations avec le droit de l'Etat sont ainsi devenus incontournables pour les organisations indiennes engagées dans un nouveau genre de projet politique. Malgré les ambiguïtés et les zones d'ombres du texte constitutionnel, ces enjeux contraignent également les autres acteurs institutionnels de la société équatorienne. La délimitation normative que présuppose la garantie constitutionnelle débouche sur la question suivante : "peut-on différencier les droits autochtones amazoniens du droit équatorien officiel en fonction de critères culturels ?». Nous nous attacherons à l'examen de cette question culturo-juridique.

Cet article nous permettra de démontrer, sur la base de l'analyse de deux conflits concrets tirés des quotidiens shiwiar et runa, qu'il est difficile de répondre à cette question de manière affirmative (1) ou négative (2). Pour sortir de ce faux-débat, on interrogera la formulation de cette distinction entre droits autochtones et droit officiel et ce qu'elle suppose en terme de constitutionnalisation ${ }^{8}$ (3). Cette porte de sortie invite à penser autrement les relations entre « droits » et « cultures » en Amazonie équatorienne (4).

\section{«Oui ! : une réponse limitée}

A première vue, il est évident que les normes des groupes runa et shiwiar relèvent d'un autre registre culturel que celui du droit - étatique - équatorien. L'argument s'impose car les contextes culturels des institutions étatiques et des familles autochtones vivant en forêt sont clairement différenciables. Le fonctionnement d'un ministère de Quito ou d'une juridiction de Puyo ne repose pas sur les mêmes mécanismes que la prise de décision au sein d'une famille vivant en forêt, loin des préoccupations bureaucratiques des premières.

7. Les droits collectifs indigènes furent reconnus par la Constitution presque au même moment où l'Equateur signait la Convention n ${ }^{\circ} 169$ de l'OIT. Le texte de la Constitution s'en inspire largement. Cette reconnaissance fut célébrée comme une victoire par les organisations indigènes du pays. Un imposant article, l'article 84 divisé en quinze paragraphes, énumère les droits collectifs reconnus aux peuples indigènes. D'autres articles, dispersés dans le corps de la Constitution, en détaillent quelques-uns (division administrative et exercice de la justice indigène). La Constitution équatorienne déclare, depuis 1998, que «les peuples indigènes qui s'autodéfinissent comme des nationalités d'origine ancestrale, ainsi que les peuples afroaméricains, font partie de l'Etat équatorien, unique et indivisible » (art. 84 alinéa 1). On remarque que cette formulation implique une inclusion des peuples indigènes dans un Etat unique et indivisible [font partie] tout en soulignant leur origine « extérieure » [les racines ancestrales]. La rédaction de l'article 84 reconnaît et garantit l'exercice des droits collectifs dans le respect du reste de la Constitution et des lois. Cette clause de sauvegarde et le reste des dispositions constitutionnelles empêchent de donner une interprétation et une application univoque aux droits collectifs autochtones et les privent d'une potentielle effectivité. La nature constitutionnelle du texte est rendue inopérante en raison de techniques de rédaction juridique dont on peut s'étonner qu'elles n'aient pas suscité plus d'opposition.

8. Ces deux cas se déroulent après 1998. Les commentateurs et informateurs locaux se sont référés à un moment ou un autre du travail d'interprétation aux «droits collectifs » reconnus par la Constitution de 1998. 
Ces réalités distinctes produisent des normativités dont l'ampleur, les formes et le genre de rationalité diffèrent.

Cette différence constitue le point de départ de la réflexion des spécialistes de droits autochtones consacrée aux questions de compatibilité entre les normes autochtones et le droit étatique. Ces spécialistes conceptualisent des réalités culturelles et juridiques, discernables et relativement stables. Ce postulat de travail est en quelque sorte conforté par l'existence de travaux ethnographiques, nombreux et richement détaillés, portant sur les groupes amazoniens de cette région (Descola 1986, 1993b; Guzman 1997; Reeve 1988; Taylor 1993a, 1993b, 2003; Whitten 1976, 1985). Les illustrations circonstanciées des logiques locales qu'ils contiennent peuvent être utilisées dans ce sens. Mais nous verrons qu'il n'est pas si évident de transformer ces constats ethnologiques en principe juridique.

\section{L'évidence des différences}

Le récit suivant illustre la spécificité et l'acuité de logiques locales :

Franklin, un homme né dans une famille runa établie dans une région proche de Puyo prend Florentina, une jeune femme shiwiar, comme épouse et va vivre dans la communauté de cette dernière. Il y exerce le métier de maître d'école et jouit d'une visibilité sociale. Le jeune couple vit quelques années auprès de leurs (beaux-) parents shiwiar. Mais ils quittent de manière temporaire cette région après avoir eu leur premier enfant. Ils s'installent dans la petite ville de Puyo où sont basées les organisations indigènes de la région. Franklin travaille à différents titres dans deux d'entre elles. Leur deuxième enfant naît pendant cette période. Le nourrisson est pris à plusieurs reprises de fortes fièvres. Les jeunes parents se rendent à l'hôpital et consultent un chamane, oncle de Franklin. Rien n'y fait. Le petit garçon décède avant d'avoir accompli un an. Après avoir enterré le corps dans la région des grands-parents paternels ["sa petite maison" comme évoque tristement le père endeuillé], Franklin consulte trois chamanes. Il cherche à déterminer la cause de la mort de l'enfant. A l'issue de ces consultations, Franklin explique qu'il "sait» que son fils a été "chamanisé » [chamaneado] par un vieil homme shiwiar dont il affirme que "tout le monde sait qu'il est chamane ». Ce chamane vit dans une communauté voisine de l'endroit où sont établis leur famille shiwiar avec laquelle le couple continue à s'identifier. Lors de l'installation de Florentina et de Franklin, cet homme avait prononcé publiquement une phrase dont la clarté et les implications ne sont pas tous apparents.

"Quand la femme mourra, il [Franklin] devra retourner d'où il vient ».

Pour Franklin la phrase ne fait l'objet d'aucun doute interprétatif. Le décès de son fils prouve que ces propos étaient des menaces de mort. La mort de son enfant est lue à la lumière de ces propos dont le caractère menaçant est établi de façon circulaire. A la suite des séances de diagnostic, Franklin rédige une lettre avec l'aide du frère de son épouse, solidaire de ce malheur familial. Ils font savoir au vieil homme que tout ce qui devrait arriver à la famille de Franklin dans le futur fera l'objet "d'investigation » [i.e. Franklin ira "faire voir" ces évènements par d'autres chamanes]. Selon Franklin, sa lettre ne laisse planer aucun doute et le vieil homme "sait " maintenant qu'une nouvelle attaque de sa part entraînera une réplique mortelle. La lettre est transmise par le fonctionnaire chargé des missions de police - le teniente polético - de la petite juridiction amazonienne de Rio Corrientes où le conflit a débuté. La question n'est pas évoquée publiquement et les hommes, lorsqu'ils se croisent dans le cadre d'activités et des festivités politiques, ne l'abordent pas. Mais un nouveau "malheur » pourrait déclencher une contre-offense. 
Des logiques normatives locales émergent du récit de Franklin. Les « normes » et les procédures auxquelles il se réfère sont en prise directe avec un paradigme chamanique. Le caractère constitutif de ce paradigme dans les subjectivités amazoniennes fait l'objet de l'attention de nombreux anthropologues (Albert 1993; Brown 1993; Chaumeil 2000; Conklin 2002; Gow 1990; Viveiros de Castro 1986; Whitten 1985 entre autres). Il n'est donc guère surprenant qu'il apparaisse dans le déroulement de ce conflit.

La structure normative de ce récit correspond assez distinctement à une narrativité vindicatoire $^{9}$. On remarquera que l'énonciation de règle ne précède pas la description des faits dans le récit de Franklin. Au contraire, c'est la description factuelle, opérée par le narrateur, qui permet d'identifier implicitement une règle générale : la réparation ou la compensation d'un dommage affectant le bien-être d'un groupe par une action « réciproque ».

Toutefois cette règle n'est absolument pas explicitée dans la narration. La cohérence de l'interprétation de Franklin semble ne pas dépendre de l'identification explicite d'une règle. Elle se déploie à un tout autre niveau. Dans ce récit, la règle est littéralement contenue dans l'établissement des faits.

On peut en déduire que circonstances et subjectivités en conflit peuvent affecter et modeler la structure normative. Le normatif prend ici une allure plus sociale et moins typologique que dans des systèmes juridiques écrits et institutionnalisé de manière formelle. Le statut implicite des propositions normatives a fait l'objet d'une attention particulière dans le développement de l'anthropologie juridique (Comaroff et Roberts 1981; Greenhouse 1985). Il caractérise de nombreux ensembles normatifs que l'on peut opposer sur ce point aux droits modernes d'origine continentale - et à leurs accents légalistes bien plus explicites ${ }^{10}$. Arrêtons-nous un instant sur la structuration de ce conflit pour en dégager les éléments culturels importants.

\section{Structuration culturelle du conflit}

La procédure chamanique fonctionne comme un procédé qui établit ou valide les faits de manière radicale. Le diagnostic des trois chamanes constitue un point d'inflexion dans le récit de Franklin. C'est à partir de ce moment que la signification des évènements s'impose en bloc pour une partie au conflit. Les «faits » litigieux ainsi formalisés déterminent dans un même mouvement la nature de la mort et les identités collectives de la victime et de l'agresseur. Ils organisent le conflit.

9. Ce terme est emprunté à l'œuvre de Raymond Verdier à qui l'anthropologie juridique doit une théorie stimulante de la vengeance en tant que procédé normatif. Pour cet auteur, « au plan de la communication sociale, la vengeance est un rapport d'échange bilatéral résultant de la réversion de l'offense et de la permutation des rôles de l'offenseur et de l'offensé suscitant une contre-offense, la relation s'inverse, l'offensé devient offenseur et vice versa » (Verdier 1980 : 14).

10. Il faut évidemment nuancer cette opposition qui procède de l'idéal-type wébérien. Les usages sociaux des droits « officiels » donnent également lieu à des situations dans lesquelles les propositions normatives restent implicites. Mais la propension explicite et classificatoire des droits officiels reste indéniable. Les droits étatiques - surtout d'origine civiliste - restent idéalement structurés par la détermination et l'explicitation des propositions normatives. 
Le cœur du conflit ne porte donc pas sur l'existence, l'interprétation ou l'application d'une règle imprimant une rationalité classificatoire ou syllogistique ${ }^{11}$ aux évènements. L'enjeu repose presque entièrement dans l'opposition de réalités factuelles contradictoires révélées par des procédures spécialisées. L'issue de ce genre de litige est donc liée à la réception et à la mobilisation sociale que suscite la description de ces «réalités » différentes. Le stade dans lequel l'histoire de Franklin se trouve au moment de son récit est celui de latence. Le conflit a acquis suffisamment de consistance sociale pour pouvoir se distinguer des autres aléas de la vie quotidienne. Mais sa direction reste incertaine.

En effet, la version de Franklin dévoile l'existence d'un conflit qui acquiert progressivement une forme sociale spécifique. Sa version est sanctionnée par un diagnostic chamanique qui, à l'instar des procédures « juridiques », imprime une spécificité sociale au conflit. Le différend est suffisamment structurant pour acquérir une valeur heuristique au sein du groupe familial fédéré autour de Franklin. Les tensions avec des dirigeants proches du chamane malfaisant sont analysées et commentées par le groupe solidaire de Franklin par référence au premier incident.

De leur côté, le chamane et sa famille ne contestent pas ces " faits » publiquement. A ce stade, l'affaire fait l'objet d'une diffusion et d'une publicité restreinte qui coïncide avec un degré médian de conflictualité sociale. On peut penser que plus cette version aura circulé et aura été socialement confortée et diffusée par d'autres familles ou d'autres évènements, plus le conflit deviendra " public» et grave. L'opposition entre les parties se sédimentera et l'exigence d'une « compensation » viendra s'imposer aux membres de l'une d'entre elle de manière contraignante.

Pour l'autre partie, celle du chamane, l'établissement de ces faits n'appelle aucune réponse. Les faits pertinents pour cet adversaire sont probablement d'une autre nature. Les menaces et le rejet exprimés par le vieil homme, l'ont été au moment de la formation du couple. Les règles en cause ne sont à nouveau pas explicitées dans la phrase dont se rappelle vivement Franklin. La « menace » identifiée a posteriori n'est pas le fruit de son imagination. Elle exprime et formule un « reproche».

Elle communique un message relatif à l'inadéquation sociale de Franklin qui devra « retourner d'où il vient». Il est clair que cette nouvelle alliance et la résidence du jeune couple pose problème au chamane. La phrase du vieil homme est une contestation publique de la résidence territoriale du couple - voire même de la légitimité de celui$\mathrm{ci}^{12}$. Le couple étant inacceptable pour les uns, sa contestation est offensante pour les autres. Ce n'est pas par hasard, que dans l'interprétation de Franklin, elle «menace» littéralement sa famille.

La construction culturelle de l'offense cristallise des logiques d'opposition dans un paradigme chamanique. La première offense, la contestation de la résidence, se matérialise dans l'attaque du chamane et la mort de l'enfant. Le diagnostic chamanique en établit la

11. Le syllogisme judiciaire est souvent présenté comme une figure idéalisée des droits officiels civilistes. Il fonctionne en trois étapes. L'application d'une majeure (la règle) à une mineure (les « faits ») permet de déduire la décision.

12. Franklin n'est pas un enfant du pays. Très lointain cousin, il a grandi à bonne distance des parents de Florentina. A aucun moment de son récit, il ne fait référence aux règles de l'alliance des familles shiwiar ou runa. Il se contentera d'expliquer qu'un homme préparé (en se référant à son statut de maître d'école) suscite toujours des jalousies. 
réalité sociale. Cette intervention opère de manière temporelle et spatiale et télescope les lectures rétrospectives des évènements en les organisant sur un même plan.

On comprend bien que le chamanisme et la régulation vindicatoire qui le caractérise dans le cas que nous évoquons, se décrivent mal en termes de système d'autorités fixes et prédéterminées. Ils ne «fonctionnent » pas de cette manière. Comme l'écrit R. Verdier :

Qu'elle soit exercée par des acteurs individuels ou collectifs, la vengeance dans le système vindicatoire est essentiellement un phénomène intergroupal : elle oppose des groupes qui en s'opposant manifestent leur unité propre dans le jeu de l'offense et de la contre-offense (Verdier $1980: 18$ ).

Chamanisme et logique vindicatoire expriment des règles plutôt qu'ils ne les «appliquent». Une lecture en termes structuro-fonctionnalistes permet de saisir le caractère central de ces processus dans la (re)production des groupes de solidarité. On peut reprocher aux perspectives structuro-fonctionnalistes un certain tautologisme social. Cette critique mise à part, le mérite de telles approches est bien de mettre à jour les spécificités locales des formes d'expressions et de « résolution» de conflits ${ }^{13}$.

L'analyse de notre question de départ nécessite que l'on retienne certains éléments. La cohérence de la logique vindicatoire qui se déploie dans le cas examiné se démarque nettement de celle que les droits étatiques prétendent mettre en œuvre. Sans entrer dans le débat relatif au statut de la "peine » dans le droit pénal, il faut insister sur l'absence totale de pertinence de la distinction entre droit pénal et droit privé dans le déroulement de ce conflit. Elle n'a aucun « sens » pour les acteurs en présence.

Les règles d'alliance et de résidence qui traversent ce conflit ne sont pas appréhendées dans une forme et un contenu comparables à ceux des règles sur le mariage et la liberté d'établissement des personnes du code civil équatorien. Les attaques chamaniques sont pour leur part des réalités sociales complètement ignorées des catégories juridiques équatoriennes. Elles soulèvent des problèmes de preuve pratiquement insolubles pour un juge équatorien.

Qui plus est, il reste difficile d'envisager l'intervention chamanique comme procédant d'une autorité judiciaire. Les expertises chamaniques dépendent des parties en présence et ne reposent sur aucun systématisme ni hiérarchie, au contraire de l'organisation judiciaire.

Il semble donc que notre question initiale se résolve sans peine par l'affirmative. Comme on pouvait le pressentir, on peut identifier des logiques normatives locales suffisamment caractéristiques et culturellement significatives pour les distinguer du droit pénal et civil étatique. Voilà sans doute pourquoi certains spécialistes se concentrent sur l'établissement de principes de compatibilité entre ces ensembles différenciés.

13. On n'en déduira toutefois pas, sous peine d'essentialisme culturel, que les normativités autochtones soient réductibles à cette logique vindicatoire. Elles débordent la manifestation du vindicatoire qui structure les « crises » bien plus que le train-train quotidien. 


\section{La lettre : une scorie ethnographique ? - l'appropriation du droit officiel}

Une analyse qui en resterait là se coupe pourtant de tout un pan du récit. On a constaté que les logiques vindicatoires se déploient sans que l'intervention d'autorités centralisées ne soit nécessaire. Mais il faut cependant analyser le statut de la «lettre» dans le déroulement de notre affaire. Elle est un opérateur social dans l'évolution du conflit. La transmission de la lettre inverse le sens de l'offense et signale que le conflit a atteint un certain degré de conflictualité sociale. Elle est l'instrument d'un échange de menaces. Paradoxalement, cet échange est garanti par une autorité étatique. Les distinctions entre normativités autochtones et officielles s'estompent, les principes de "compatibilité » également.

Franklin pourrait très bien envoyer la lettre par ses propres moyens. Mais il la confie à l'« autorité publique » de la région, le teniente polético. Il s'agit d'un fonctionnaire payé et nommé par le ministère de l'intérieur. Depuis 1997, le droit équatorien a été modifié pour confier aux assemblées des "communautés » le soin de choisir ce fonctionnaire dans les juridictions à prédominance indigène. Dans celle de Rio Corrientes, les tenientes poléticos qui ont exercé ces dernières années sont des résidents - masculins - shiwiar. Ils expliquent que leur rôle est de « conseiller» les familles et de faire appliquer la loi. Ils écrivent des rapports à leur supérieur, le gouverneur de la province de Pastaza, établit à Puyo. Dans ces rapports, l'explication de la Constitution aux communautés est évoquée de la même manière qu'un avertissement adressé à un « mauvais » chamane.

Le rôle du teniente dans ce conflit illustre l'imbrication de différentes logiques. Le recours au tiers et à la puissance d'un Etat " indigénisé » ne peut être évacué du récit de Franklin. Ici comme ailleurs, le déploiement des logiques vindicatoires s'accommode de la présence d'éléments d'autres registres institutionnels - qui les décrivent pourtant mal - : l'« autorité » et le « papier $»^{14}$.

On ne considérera toutefois pas que les normativités autochtones perdent de ce fait leur spécificité, puisque le recours aux «autorités étatiques » est motivé par des logiques locales. En ce sens, on peut parler d' «appropriations » du droit officiel par des acteurs autochtones. Ces processus intègrent bien plus les formes du droit officiel que ses contenus ou logiques. Dans ces usages symboliques, le contenu des règles du droit officiel n'a guère d'intérêt performatif. Les logiques locales instrumentalisent les pouvoirs officiels au profit des parties qui s'affrontent. Mais ces instrumentalisations et appropriations ont évidemment un coût social : elles viennent renforcer la légitimité des institutions qu'elles sollicitent.

14. On constate d'ailleurs que dans le Pastaza, les logiques autochtones les intègrent partiellement depuis longtemps. Les premiers ethnographes de cette région l'illustraient déjà « Thus, for instance, the Jibaro chief Nayapi on the Pastaza, and the old Canelos chief Palati on the Bobonaza had for many years been enemies and had sent menacing messages to each other. In the family of Nayapi within a comparatively short time several deaths took place [...]. Nayapi said that his enemy Palati was the cause of all these deaths by systematically letting off his magic arrows against Nayapi and his family. Palati, again by no means denied that this was so, but, on the contrary, confirmed it, meaningly announcing that he would, gradually exterminate Nayapi's whole family. The latter was seized with wrath and desire for revenge, and certainly would have wreaked a terrible vengeance upon his enemy if regard for the Catholic monks, under whose protection Palati stood, had not made him abstain from carrying it out » (Karsten 1935 : 276-277). 
Il est évident que ces « intégrations » peuvent influer, à leur tour, sur la reproduction $\mathrm{du}$ « système vindicatoire » et sur l'équilibre politique et social qu'il entretient ${ }^{15}$. Les pratiques des fonctionnaires indigènes ${ }^{16}$ illustrent bien cette ambiguïté. D'ailleurs, l'effectivité de leurs « interventions » dépend fortement de leurs capacités personnelles et de leur pouvoir de conviction - au contraire du fonctionnaire lambda dont le pouvoir découle d'une puissance publique idéalisée. Elle est déterminée par l'état de leurs réseaux et supports familiaux. Le teniente qui est intervenu dans le cas qui nous occupe avouait sans sourciller qu'il ne s'aventurait pas dans une partie de sa juridiction où vit un noyau de familles impliquées dans un meurtre affectant la sienne. Il considérait simplement que cette région n'était pas dans sa juridiction ... contrairement aux cartes officielles. La géographie institutionnelle change donc en fonction de la personne qui exerce l'office public sans que cela fasse l'objet de l'attention d'autorités supérieures évanescentes.

Une réponse positive à notre question initiale ferait l'impasse sur la mobilisation du droit officiel qu'opèrent des acteurs autochtones animés par des logiques de pouvoir locales. Il est donc difficile de répondre « oui ». Mais faut-il répondre par la négative et conclure qu'il est impossible de distinguer les logiques locales trop imbriquées dans un paradigme officiel ? Dans le point suivant, nous envisagerons une explication en terme d'acculturation juridique et culturelle qui renoncerait à identifier des réalités normatives différentes. C'est l'analyse d'un autre cas qui nous permettra d'explorer les limites d'une telle position.

\section{« Non!» : les limites d'une analyse en termes d'acculturation}

Une histoire contenue dans les archives d'une organisation runa illustre l'importance croissante des catégories juridiques du droit officiel dans l'organisation des conflits entre familles runa. Elle pose la question de l'acculturation juridique.

La propriété : une relation juridique importée

Le "président» d'une communauté runa du Bobonaza écrit une lettre à celui de l'organisation régionale runa basée à Puyo. Il s'adresse à ce président, précise-t-il, en tant qu' "autorité » et l'informe des menaces de mort proférées par José, membre d'une communauté de l'aval, à l'encontre d'un chamane de sa communauté, Gustavo. José a insulté plusieurs proches et voisins du chamane venus récolter des feuilles d'une espèce de palmiers - relativement dispersée dans la forêt mais très prisée pour la toiture des maisons - dans les environs de son foyer. Dans cette lettre, le président commence par rappeler que la personnalité juridique de sa communauté a été légalement accordée ${ }^{17}$. Il explique ensuite "que les territoires globaux sont délimités à M. qui se trouvent

15. L'introduction des armes à feu en Amazonie équatorienne, « innovation technique » s'il en est, a eu des conséquences désastreuses sur les vendetta et leur régulation très perceptibles dès les années 50 (Descola1993b :177, 187).

16. Le teniente polético dans le cas évoqué était un maître d'école shiwiar de la région. Il faut noter que le recours aux autorités officielles est relativement indépendant de ces appartenances ethniques. Dans d'autres affaires, des familles runa et shiwiar n'ont pas hésité à impliquer la police nationale, les militaires ou des fonctionnaires de Puyo et de Quito.

17. Une rapide recherche juridique invalide cette affirmation. Mais l'auteur de la lettre est suffisamment convaincu de son importance pour l'utiliser comme argument. 
quelques 6 kilomètres en contrebas » de la communauté où ont été proférées les menaces. Il en conclut que " ceci veut dire que les membres de [sa] communauté peuvent transiter librement dans tout ce territoire, ainsi que profiter de ses ressources naturelles ». Selon lui, ces derniers récoltaient ces feuilles "comme l'ont fait pendant des centaines d'années nos ancêtres grands parents et parents ${ }^{18}$.

La référence aux titres de propriété collective obtenus en 1992 et à un " territoire » que l'on peut s'approprier et délimiter systématiquement, imprime à ce conflit les caractéristiques de la "propriété ». Or, ce ne sont pas dans ces termes que les ethnologues décrivent les relations que les familles indigènes de cette région maintiennent avec leur environnement. Tous évoquent des territorialités rhizomiques et multiples. Les familles du Bobonaza se seraient à ce point « acculturées » qu'elles auraient « adopté » le modèle de la propriété ${ }^{19}$ dans leurs relations sociales ? Une telle analyse n'a pas que des conséquences ethnologiques. Cette « adoption » pourrait être utilisée comme un argument pour refuser la protection constitutionnelle à des peuples ne démontrant pas leur spécificité juridicoculturelle.

On pourrait rétorquer qu'il s'agit en fait d'un problème de traduction, une question classique en anthropologie. On sait depuis longtemps que « nos » catégories de pensée - y compris juridiques - sont souvent inadéquates pour « traduire » la réalité d'autres cultures. Mais dans cette affaire, il ne s'agit pas d'un problème de traduction ethnographique. L'énoncé n'est pas adressé à un anthropologue, il est destiné à circuler entre familles voisines en conflit. La lettre est écrite en espagnol par le président de la communauté runa. " L'indigène » se « tromperait » de registre ?

On ne peut guère douter de l'importance sociale qu'accorde ce président aux limites de la propriété de sa communauté. Mais il serait simpliste de conclure qu'il «trahit» sa propre culture et «ses » catégories de pensée et d'action en intégrant ces termes juridiquement connotés. En effet, que reste-t-il de la «culture » au niveau analytique, si on exclut de son champ les capacités personnelles et collectives des acteurs pour organiser et se représenter le réel ? D'autant plus que le reste de la lettre du président témoigne de la vigueur des logiques locales présidant ses réappropriations d'éléments juridiques.

\section{La vigueur des logiques locales}

Dans le reste de la lettre, le président se plaint des menaces de mort proférées contre un autre chamane de sa communauté. Il écrit, ce « fait s'inscrit dans une vague de malaises et de harcèlement qui a surgi entre les deux communautés en raison de deux professeurs manipulateurs ». Les deux chamanes menacés publiquement, sont des " anciens sages, chamanes de la communauté ». «Ils font partie des personnes peu nombreuses qui

18. On appréciera évidemment la profondeur généalogique de l'argument. La référence " ancestrale » est sans doute inspirée du vocabulaire constitutionnel et de la Convention 169 de l'OIT. Sa signification ne coïncide pas avec le concept anthropologique d'ancestralité utilisée dans la typologie des systèmes de parenté et la description du culte des « ancêtres " associés à une organisation lignagère. Ici l'ancestralité correspond plus à un registre d'argumentation qu'à la description d'un système de parenté. Elle sert de critère « immémorial » de légitimité (Ingold $2000: 133$ ).

19. Le principe de l'appropriation et du contrôle d'une chose par un sujet est déterminant dans les modèles de propriété individuelle et collective. Il est difficilement conciliable avec les descriptions ethnologiques de la région. 
possèdent la science millénaire de la médecine indigène, connus et appréciés à l'intérieur et à l'extérieur du pays », affirme le président. Il range les menaces et les manigances des maîtres d'école ${ }^{20}$ exerçant dans ces communautés voisines dans la catégorie d'agression et d'offense. Il complète sa longue série de récriminations en écrivant qu'on suspecte José d'un assassinat. «Le monsieur mentionné ne jouit pas de notre confiance, ni de celle de sa propre communauté ».

Le contexte évoqué par cette lettre découvre au fur et à mesure une série de « problèmes " : des menaces, des contestations relatives à l'accès aux ressources d'une région voisine et un climat d'accusation et de tensions entre groupes de maisonnées. La missive se clôt par une déclaration claire «ma communauté est respectueuse de ses gens, des autres communautés et des différences des autres cultures, mais c'est aussi un peuple très digne qui n'a jamais permis et qui ne permettra jamais que des indésirables outragent et tachent sa dignité et ses droits. (..) Afin de ne pas altérer la convivialité pacifique entre ces deux communautés par des affrontements non nécessaires, nous sollicitons des autorités de Pastaza qu'elles réalisent de manière urgente une investigation exhaustive et que soit imposée une sanction sévère ».

Une structure normative vindicatoire, comparable à celle que nous avons analysée dans le premier cas, alimente le déroulement du conflit tel qu'en rend compte la lettre de ce président. Sa plainte est difficilement réductible au seul langage de la propriété. L'expression du conflit dépasse également les distinctions entre le droit pénal et le droit privé qui organisent le droit étatique.

La lettre exprime et publie l'atteinte aux droits de la communauté de manière claire. Elle « informe » et donc transforme l'extension socio-institutionnelle du conflit. Elle constitue une réplique menaçante aux offenses perpétrées par José et par les maîtres d'écoles qui ont porté atteinte à l'honneur social de la " communauté ». Le Président, fait savoir que sa communauté ne se laissera pas humilier et entend rétablir son honneur. Les démarcations entre groupes vindicatoires sont donc maintenues et entretenues. Quant aux agresseurs, ils sont « officiellement » tenus pour personae non gratae. La lettre consolide une « relation d'adversité » (Verdier 1980). Comme dans le cas précédent, la lettre paraofficielle est chargée d'effet.

\section{Les significations attachées à la « réappropriation » du registre officiel}

C'est pour établir la réalité de l'outrage que le président intègre des éléments du droit formel. Il entend fonder la légitimité du droit d'accès aux feuillages précieux en recourant à plusieurs registres. Il s'appuie sur un argument immémorial « depuis des centaines d'années... ». Mais il invoque surtout le titre de propriété délivré par l'Etat ${ }^{21}$.

Ces titres ont été acquis à l'issue d'une mobilisation impressionnante des familles indiennes de la province par l'OPIP, une organisation indienne, à l'époque très active. Ces titres de propriété sont malheureusement inadéquats et traversés d'incohérences juridiques. Ils ont été adoptés sur base de la loi des « terrains vagues », avant la réforme constitutionnelle de 1998.

20. Il s'agit de jeunes hommes d'autres communautés runa et achuar.

21. En 1992 par l'Institut Equatorien de la Réforme Agraire et de la Colonisation (IERAC) devenu depuis lors Institut National de Développement Agraire (INDA). 
Cette loi institue la fiction de la propriété de l'Etat sur les «terrains vagues ». Cet instrument juridique particulièrement contestable au regard de la Constitution de 1998 fut appliqué aux territoires amazoniens. Les titres établissent donc, par défaut, une propriété «publique » contestée. Ces titres sont donc marqués d'une ambiguïté profonde quant à l'origine de « droits ».

En ce qui concerne la délimitation des prérogatives des titulaires, les titres recourent au critère de « limites ancestrales » tout en indiquant qu'il s'agit de « limites à tracer ». On ne peut donc pas savoir si chacune des ces « propriétés » est déduite d'un accord présidant la délimitation ou si la délimitation sert à « révéler» des prérogatives préétablies.

Ces confusions se greffent aux caractéristiques des géographies indigènes qui incorporent des processus discontinus et concurrents. La constitution de ces géographies fait en général l'économie de procédés d'abstraction et de centralisation. Le recours à une géographie standardisée et fondée sur un postulat cognitif unidimensionnel et systématique ne peut être qu'imparfaitement combinée avec ces géographies variables et bien plus interactionnistes. Ce n'est donc pas étonnant que les titres de propriété reflètent la complexité de ces réalités de manière insatisfaisante.

De quelle manière la règle de propriété collective intervient dans l'argumentaire du président ? Dans ce texte culturel, la « propriété collective » est moins utilisée comme une règle dont on chercherait l'application qu'elle n'exprime, dans un nouveau lexique, un 《 fait » perçu comme préexistant. La « propriété » y apparaît plus comme une conséquence lexicale des délimitations politiques que comme une norme que l'on chercherait à « appliquer ». Il s'agit là d'un usage social de la « propriété » pour le moins original par rapport à la relation entre sujet et objet consacrée par le code civil équatorien.

L'importance de la dimension sociale dans cette "réappropriation » d'éléments du droit officiel est particulièrement criante si l'on s'attache au contexte dans lequel cette « règle» a progressivement été invoquée. Des titres de propriété collective ont été attribués par l'Etat équatorien dans cette zone de conflit alors que différentes familles runa utilisaient, connaissaient et se rendaient dans des parties de cette zone (Reeve 1988 : 38). Ces utilisations rendaient bien difficile la délimitation claire de droits différenciés.

Il semble que l'attribution des titres de propriété soit plus une conséquence de ces conflits et contestations entre familles que la représentation des prérogatives de ces différents groupes. La "propriété » s'intègre donc dans les relations entre groupes autochtones parce qu'elle offre une nouvelle forme de légitimité à des logiques culturelles préexistantes. Cette intégration donne une dimension sociale (relations entre voisins) à une figure juridique qui consacre pourtant une relation entre personne et objet.

Dans ce deuxième conflit, les logiques locales infusent également le recours au registre juridique « officiel ». Comme nous pensons l'avoir illustré, leur spécificité n'a toutefois pas diminué. Mais il faut renoncer à identifier les réalités stables que suppose la reconnaissance constitutionnelle des droits autochtones.

Ala suite de l'analyse de ces deux conflits, il est difficile de conclure que des « systèmes » normatifs amazoniens - dont on aperçoit le caractère ouvert - aient radicalement disparu. Ils recyclent certaines formes juridiques et provoquent leur propre transformation. De leur côté, les logiques locales peuvent également être instrumentalisées par une panoplie d'acteurs. Les compagnies pétrolières et d'autres commerçants ont tout intérêt à alimenter des honneurs amazoniens fracturés.

C'est précisément cette imbrication qui est évacuée lorsque l'on imagine des « peuples autochtones » et des « sociétés nationales » étanches - partout et en tout temps. 
La manière dont est posée la question initiale débouche donc sur une impasse théorique et méthodologique. Si l'on ne peut répondre - ni oui, ni non - à notre question de départ, c'est probablement qu'elle mériterait d'être posée autrement.

\section{Une question mal posée}

Dans une affaire très difficile, les « autorités » d'une communauté runa dans laquelle un meurtre s'était déroulé actèrent la condamnation à mort du meurtrier en désignant la famille de la victime comme exécuteur ${ }^{22}$. Entre peine et vengeance, le statut de l'intervention des «autorités » était pour le moins ambigu. A nouveau, elle témoigne d'une imbrication de répertoires qui semble s'être accentuée avec l'industrialisation pétrolière de la région - et que la reconnaissance constitutionnelle n'est pas parvenue à enrayer, au contraire. Le malaise $\mathrm{e}^{23}$ et la peur d'être accusés de «sauvagisme » à un moment crucial dans leur lutte contre un projet d'exploration pétrolière décidèrent finalement les dirigeants de la communauté à convaincre l'assemblée de s'en remettre à la police nationale.

Au lieu de les féliciter, le procureur chargé de l'affaire s'en étonna publiquement. « Les indiens n'ont-ils pas leur propre manière de régler ce genre d'affaire ? », s'exclama-t-il ! Il ne montrait aucun désir de se saisir d'un meurtre qui ne lui causerait qu'une surcharge de travail. C'est à peine s'il pouvait localiser la communauté en question. La distinction culturelle des normativités sert en pratique de multiples agendas.

La famille de la victime constituée en accusation particulière ${ }^{24}$ fut très vite déboutée de l'instance. Elle ne s'était pas présentée à l'audience au jour fixé ! Le frère du défunt était arrivé en retard à la ville de Puyo après une remontée de la rivière en pirogue d'une journée. Les dirigeants qui avaient suivi l'affaire furent considérés comme incompétents par les familles de la communauté. Ils avaient perdu le contrôle de la « situation » et les rumeurs les plus folles couraient dans tous les foyers. Elles prétendent que le meurtrier, condamné à un peu plus d'un an de prison, cherche à se venger.

Dans cette affaire, l'imbrication des logiques affecte le bien-être du groupe tant au moment du meurtre qu'à celui de son «jugement». Le malaise crée par le meurtre est transféré aux « dirigeants » chargé d'y remédier. Ces derniers sont dans une situation relativement insoluble. Ils doivent représenter des intérêts supérieurs - communs - aux deux familles que la mort oppose. Ces dirigeants membres de la communauté ne peuvent dénier la pertinence «culturelle » d'une logique d'honneur. Mais ils ne parviennent heureusement - pas à la concilier avec la structure « formelle » de pouvoir à laquelle ils empruntent. Conscients de la représentation extrêmement négative attachée aux pratiques de vendetta, ils en défèrent aux autorités étatiques.

22. Ici, comme dans les deux autres cas, une analyse en terme de compatibilité tourne vite court. La peine de mort étant clairement prohibée par la législation équatorienne et les instruments internationaux protecteurs des droits de l'homme.

23. Les dirigeants impliqués dans le cas l'exprimaient en soulignant que c'était la première fois qu'une « affaire » pareille se déroulait « chez eux ». Cette insistance sur la «nouveauté » affecte sans doute plus le statut de leur propre intervention, en tant qu'autorité d'une communauté définie a priori, que celle de l'occurrence d'un meurtre.

24. Il s'agit d'une possibilité de la procédure criminelle ouverte aux victimes d'une infraction pénale existant dans le droit équatorien. 
La distinction de droits autochtones de celui de l'Etat sur base de critères culturels, ne leur offre aucune «solution » pratique. Au bout du compte, la vigueur des processus de réappropriation parachève l'érosion du pouvoir des groupes autochtones. La position des dirigeants, incapables de gérer les conflits dans ces termes contradictoires, se fragilise dans un contexte houleux de négociations pétrolières. Les conflits « suspendus » alimentent les fractures entre groupes et prennent une dimension aiguë dans un cadre politique plus vaste.

Pour sortir de l'impasse théorique et méthodologique sur laquelle débouche la délimitation de droits en fonction de critères culturels, il faut en dégager l'origine. Ce sont les termes de la Constitution négociée entre les acteurs politiques équatoriens qui $\mathrm{y}$ font référence. Les institutions sociales et l'identité culturelle des peuples indigènes sont textuellement reconnues dans ces articles de la Constitution qui engage l'Etat «à renforcer l'identité et les traditions dans les domaines spirituel, culturel, linguistique, social, politique et économique » de peuples indigènes et afro-équatoriens et à protéger « leur pratique de gestion [manejo] de la biodiversité ».

L'article 191 prévoit que « les autorités des peuples indigènes exerceront des fonctions de justice, en appliquant leurs propres normes et procédures pour la solution des conflits internes en conformité avec leur coutume ou droit coutumier, dans les cas où il n'y a pas de contradiction avec la Constitution et les lois »".

L'article 224 annonce l'institutionnalisation, déléguée au législateur, de circonscriptions territoriales indigènes et afro-équatoriennes dans le cadre du régime territorial administratif - dénommé « seccional»- du droit public équatorien. La possession ancestrale des terres communautaires est également garantie par l'article 84. Il est précisé que cette possession devra être légalisée - et protégée par un titre de propriété - gratuitement, par l'Etat (article 84 alinéa 3). La Constitution déclare imprescriptible, inaliénable et indivisible la propriété indigène des terres communautaires tout en aménageant la possibilité pour l'Etat de les déclarer d'utilité publique.

Dans chacun de ces articles l'existence de "systèmes culturels» - et de réalités stables - est présupposée. La question de la délimitation entre « systèmes juridiques » est elle-même tributaire de catégories culturelles et d'un imaginaire politique fonctionnaliste (Handelman 1981). L'anthropologie travaille sur la déconstruction des catégories culturelles. Sa spécificité réside dans la distinction des catégories théoriques d'analyse de celles des groupes que ces études entendent analyser. La conception systémique de droits autochtones et d'un droit étatique appartient à ce dernier, c'est-à-dire, qu'il s'agit d'une représentation « culturelle » du phénomène normatif. On pourrait donc s'en passer.

Nous pensons que la prise en considération de logiques culturelles en vue d'une meilleure justice ne devrait pas nécessairement être envisagée à partir d'oppositions entre systèmes de droit, ni d'ensembles culturels stables. Nous pensons que l'échec actuel de la constitutionnalisation équatorienne des droits autochtones est en partie lié à la réduction des dimensions culturelles dans des termes rigides.

Le moins que l'on puisse dire c'est que cette rencontre entre normes et cultures ne concrétise pas une meilleure justice. La réforme constitutionnelle paraissait « répondre » aux mobilisations et aux revendications indigènes. Mais bien qu'elle ait souvent été présentée comme une avancée dans la lutte de ces derniers, il est impossible de s'y cantonner. A plusieurs égards, cet ensemble de dispositions n'a permis aucun changement majeur pour ces peuples dans l'exercice effectif de leurs droits. 
En continuant à vouloir répondre à la question de la différenciation culturelle des normativités, on passe inévitablement sous silence des réalités conflictuelles bien plus importantes. En Amazonie équatorienne, il s'agit évidemment de la rapide industrialisation et mercantilisation de l'espace et des ressources. Les conflits qui en découlent expriment - culturellement - la transformation des structures de pouvoir en Amazonie et en Equateur.

Si l'on définit un cadre d'analyse plus large que celui de logiques locales autosuffisantes, on peut alors intégrer une nouvelle donnée : le texte constitutionnel n'est pas aussi clair qu'il n'y paraît s'il est lu dans son entièreté. Il faut passer de multiples contradictions et ambiguïtés ${ }^{25}$ sous silence pour pouvoir prétendre que l'ordre constitutionnel équatorien s'est vu fondamentalement modifié. Or c'est bien cette illusion à laquelle s'accroche tous les acteurs en présence. La proclamation de grands principes s'accompagne de la délégation au législateur de leur mise en pratique, ce qui implique, quand on y regarde de plus près des modifications transversales dans toute une série de domaines (de la fonction judiciaire au droit économique international des contrats). En 2005, on les attend toujours.

A l'heure actuelle, il ne reste aux négociateurs indiens qu'à s'arrimer à ces principes qu'ils ont arrachés de haute lutte. Ils sont coincés dans une position de nominalisme politique et ne peuvent que déplorer que les autres acteurs du jeu institutionnel n'aient pas respecté des engagements politiques et constitutionnels ambigus. Le pouvoir de cette illusion légaliste a acquis un degré suffisant pour que les dirigeants indigènes et les familles autochtones se convainquent de la nécessité de produire leur « droits coutumiers ».

Il faut souligner ici que cette configuration entre droits et cultures constitue un défi extrême pour les nouvelles " autorités " indigènes qui doivent l'affronter de manière solitaire dans un Etat au bord de la disparition. C'est sur eux que repose la responsabilité constitutionnelle, alors que les moyens et la légitimité de l'Etat continuent à leur être refusés.

Ce que ces phénomènes de juridisation (Friedman et Belley 1993) nous font perdre de vue, c'est l'ingéniosité - malgré leur relative impuissance - de ces acteurs de terrains dans la résolution des conflits. Il est à cet égard frappant de constater que la reconnaissance constitutionnelle permet aux autorités «non indigènes » de se dégager - socialement et financièrement - de ces conflits « indigènes » et donc de véritablement essayer de comprendre les logiques des acteurs et les enjeux des conflits locaux, au cas par cas.

A terme, cette nouvelle dynamique institutionnelle pourrait avoir des conséquences délétères. Le juridique et les modes de résolutions officiels ou pseudo-officiels deviennent de plus en plus importants dans les imaginaires normatifs locaux sans que la réalité des conflits ne soit investie et travaillée par le pouvoir judiciaire équatorien sous financé, ni qu'il soit effectivement possible de garantir pour les dirigeants indigènes les moyens de résoudre ces conflits.

25. La propriété de l'Etat sur les ressources du sous-sol est maintenue dans la Constitution. Elle côtoie la garantie de la possession ancestrale des terres communautaires l'air de rien. Quant aux articles « chauds », ils sont accompagnés d'une clause de sauvegarde qui précise que les droits collectifs sont garantis « dans les limites du respect de la Constitution et des lois de l'Etat équatorien ». Il semble difficile de délimiter le « pouvoir » des « autorités traditionnelles » en matière de résolution de conflits si les lois sur le pouvoir judiciaire ne sont pas elles-mêmes réformées alors que la clause de sauvegarde impose, de son côté, le respect de ces « lois». 


\section{Conclusions}

En tentant de répondre à notre question de départ, on a pu dégager des logiques locales - shiwiar et runa - spécifiques. Mais l'analyse de leur déploiement remet en cause l'existence de réalités culturelles délimitées et stables. Les registres normatifs autochtones et étatiques se superposent et coexistent dans l'expérience même des acteurs.

Toutefois, une analyse en termes d'《acculturation» rencontre vite ses limites, celles de la pensée dichotomique. Le fait que les protagonistes de ces deux cas soient des chamanes, des dirigeants d'organisations et des maîtres d'école indigènes empêche que l'on en fasse une lecture dans les termes de l'opposition fatiguée entre tradition et modernité. Les acteurs de ces affaires décodent les situations selon une logique partagée, celle de la « valeur», de la « force » et de l'honneur d'un groupe identifié par l'outrage qui le frappe, celle du vindicatoire ${ }^{26}$.

Les articulations entre registres normatifs permettent aux acteurs de construire des critères de légitimité. Ils sont utilisés dans le déroulement de leurs conflits. La nature des registres de légitimité rend la distinction culturelle entre genres de normativité inopérante et glissante. L'imbrication des arguments « formalistes » - autorité, lettre, personnalité juridique, territoires globaux, délimitation - produit, à son tour, de nouvelles représentations du territoire et des normes.

On ne peut donc pas répondre qu'il n'y a plus de « différences ». Mais on ne peut pas passer sous silence la combinaison - socialement productive - entre le droit de l'Etat et les normativités autochtones. L'introduction d'une dimension culturelle dans la technique juridique requière donc, sous peine de rester inefficaces face aux mutations des structures de pouvoir, de cesser de présupposer des réalités intangibles.

26. Les approches historiques du droit confirment que les logiques vindicatoires parviennent parfois longtemps à s'insinuer dans les termes des registres normatifs des institutions publiques (Farcy 1997; Gómez i Mestre 2003). Sans vouloir verser dans un essentialisme culturel, il semble bien que les cultures des sociétés amazoniennes du Pastaza empruntent, dans des degrés divers, à cette logique. Descola utilise son matériel de recherche auprès des Achuar pour établir la typologie des offenses, l'établissement de la relation vindicatoire, la ritualisation de la vengeance avec son éventuelle composition ainsi que le caractère proprement institutionnel de la guerre et de la vendetta dans ces pratiques. Si les Runa se défendent assez relativement d'ailleurs - de pratiquer la guerre et la vendetta, ils semblent avoir surinvesti le registre chamanique d'un caractère d'autant plus létal (Descola, 1986 : 137-139; 1993 : 172, 277-278; Whitten, 1976 : 198). L'ethos du guerrier combattant fait partie des figures que les organisations indigènes mobilisent dans leurs actions politiques qu'elles soient quichua ou jivaro. 


\section{Références bibliographiques}

Albert, Bruce, 1993. «L'Or cannibale et la chute du ciel : une critique chamanique de l'économie politique de la nature », L'Homme, 126-128 33 (2-4), pp. 349-378.

Bilhaut, Anne-Gaël, 2003. « ...Soñar, recordar y vivir con eso », Estudios Atacameños, 26, pp. 61-72.

Brown, Michael, 1993. « Facing the State, Facing the World : Amazonia's Native Leaders and the New Politics of Identity », L'Homme, 126-128, 33 (2-4), pp. 307-326.

Chaumeil, Jean-Pierre, 2000. Voir, savoir, pouvoir : le chamanisme chez les Yagua de l'Amazonie. Genève : Georg.

Comaroff, John L. \& Simon Roberts, 1981. Rules and proceses. Chicago : University of Chicago Press.

Conklin, Beth A., 2002. « Shamans versus Pirates in the Amazonian Treasure Chest », American Anthropologist, 104, 4, pp. 1050-1061.

Descola, Philippe

1986. La Nature Domestique - Symbolisme et praxis dans l'écologie des Achuar. Paris : Editions de la Maison des sciences de l'homme.

1993a. Les lances du crépuscule - Relations Jivaro Haute-Amazonie. Paris : Plon.

1993b. «Les Affinités sélectives - Alliance, guerre et prédation dans l'ensemble jivaro », L'Homme, 126-128, 33 (2-4), pp. 171-190.

Friedman, Lawrence M. et Jean-Guy Belley, 1993. « Juridicisation », in André-Jean Arnaud (dir.), Dictionnaire encyclopédique de théorie et de sociologie du droit, pp. 319-322. Paris : LGDJ.

Gow, Peter, 1990 (1996). « ¿Podía leer Sangama ? Sistemas graficos, lenguaje y shamanismo entre los Piro (Perú Oriental) », in Fernando Santos Granero (comp.) Globalización y cambio en la amazonia ecuatoriana, pp. 261-287. Quito : Abya-Yala.

Greenhouse, Carol J., 1985. « Mediation : a comparative approach », Man (N.S.) 20, pp. 90-114.

Guzman Gallegos, Maria Antonieta, 1997. Para que la yuca beba nuestra sangre-Trabajo, género y parentesco en una comunidad quichua de la Amazonia Ecuatoriana. Quito : Abya-Yala-CEDIME.

Handelman, Don, 1981, « The Idea of Bureaucratic Organization », Social Analysis, 9.

MaCDonald, Theodore, 1999. Ethnicity and Culture amidst New 'Neighbors': The Runa of Ecuador's Amazon Region. Boston : Allyn \& Bacon.

ReEve, Mary-Elizabeth, 1988. Los Quichua del Curaray - El proceso de formación de la identidad. QuitoGuayaquil : Abya Yala-Banco Central de Guayaquil.

TAYLOR, Anne-Christine

1981(1996). «La Riqueza de Dios-Los Achuar y las misiones », in F. Santos Granero (comp.) Globalización y cambio en la amazonía indígena vol. I, pp. 219-259. Quito : Abya Yala.

1993a. « Des fantômes stupéfiants - Langage et croyance dans la pensée achuar», L'Homme, 126-128, 33 (2-4), pp. 429-447.

1993b. « Les bons ennemis et les mauvais parents - Le traitement symbolique de l'alliance dans les rituels de chasse aux têtes des Shuar (Jibaro) de l'Equateur », in Elisabeth Copet-Rougier \& Françoise HéritierAugé, Les complexités de l'alliance, t. IV, pp. 73-105. Paris : Editions des archives contemporaines.

2003. « Les masques de la mémoire. Essai sur la fonction des peintures corporelles jibaro », L'Homme, 165 , pp. 223-248.

VERDIER, Raymond, 1980. «Le système vindicatoire. Esquisse théorique », in La vengeance: études d'ethnologie, d'histoire et de philosophie, tome 1, pp. 13-42. Paris : Cujas. 
Whitten, Norman E.

1976. Sacha Runa. Ethnicity and Adaptation of Ecuadorian Jungle Quichua. Urbana \& Chicago : University of Illinois Press.

1985. Sicuanga Runa. The Other Side of Development in Amazonian Ecuador. Urbana \& Chicago : University of Illinois Press.

\section{Autres références}

FARCY, Jean-Claude, 1997. «Justice, Paysannerie et Etat en France au XIX siècle », in Xavier Rousseaux et René Levy (dir.) Le pénal dans tous ses Etats - Justice, Etats et sociétés en Europe (XII ${ }^{e}-X X^{e}$ siècles), pp. 191-203. Bruxelles : Publications des FUSL.

GARí, Joseph A., 2001. «Biodiversity and indigenous agroecology in Amazonia : the Indigenous Peoples of Pastaza », Etnoecologica, 5, 7, pp. 21-37.

Gómez i Mestres, Silvia, 2003. Ordre i conflicto en terres de frontera-antropología histórica de les practiques jurídico-socials en dret penal al Vallespirm s. XIX - Thèse doctorale à l'Universitat de Barcelona.

IngOLD, Tim, 2000. « Ancestry, generation, substance, memory, land», in Tim Ingold, The Perception of the Environment, pp. 132-151. New York : Routledge.

Karsten, Rafael, 1935. The Head-Hunters of Western Amazonas : the Life and Culture of the Jibaro Indians of Eastern Ecuador and Peru. Helsingfors : Finksa vetenskaps-societeten (Commentationes humanarym litterarum [7.1]). 
VARIA 
